\title{
ESTUDO DA CINÉTICA DE DESSORÇÃO DO HIDROGÊNIO NO AÇO 9\% NÍQUEL COMO RECEBIDO E TRATADO TERMICAMENTE, APLICADO EM SISTEMAS DE FPSO*
}

Gabriel Felipe Tavares de Barros ${ }^{1}$ Wesley Pereira de Miranda ${ }^{2}$ Rafaella Martins Ribeiro ${ }^{3}$

\section{Resumo}

O presente trabalho estuda a cinética de dessorção do hidrogênio em aços $9 \%$ níquel, comercial e tratado termicamente em diferentes condições, através da técnica de Espectroscopia de Dessorção Térmica (TDS). Para a identificação dos aprisionadores de hidrogênio e a determinação da energia de ativação para a dessorção, ensaios de TDS foram realizados a 3, 5, 6, 10 e $15^{\circ} \mathrm{C} / \mathrm{min}$. Foi identificado um pico de dessorção a 124,66 kJ/mol, classificando o referido sítio aprisionador de hidrogênio como interfaces de grãos de austenita retida, cuja presença é confirmada por microscopia eletrônica e difração de raios-X.

Palavras-chave: Aço 9\% níquel; Hidrogênio; Dessorção; TDS

\section{STUDY OF HYDROGEN DESORPTION KINETICS ON 9\% NICKEL STEEL AS RECEIVED AND THERMICALLY TREATED, APPLIED IN FPSO SYSTEMS}

\begin{abstract}
The present work studies the desorption kinetics of hydrogen in commercially and treated $9 \%$ nickel steels in different conditions using the Thermal Desorption Spectroscopy (TDS) technique. For the identification of the hydrogen trappers and determination of the desorption activation energy, TDS tests were performed at 3,5 , 6,10 and $15^{\circ} \mathrm{C} / \mathrm{min}$. A desorption peak at $124.66 \mathrm{~kJ} / \mathrm{mol}$ was identified, classifying said hydrogen trapping site as retained austenite grain interfaces, the presence of which is confirmed by electron microscopy and X-ray diffraction.
\end{abstract}

Keywords: 9\% nickel steel; Hydrogen; Desorption; TDS.

1 Aluno de Graduação em Engenharia de Materiais, Laboratório de Propriedades Mecânicas, PEMM COPPE / Universidade Federal do Rio de Janeiro, RJ, Brasil.

2 Bolsista de Iniciação Científica FAPERJ, Laboratório de Propriedades Mecânicas, PEMM - COPPE/ Universidade Federal do Rio de Janeiro, RJ, Brasil.

3 Doutora em Engenharia Metalúrgica e de Materiais, Professora Adjunta, PEMM - COPPE/ Universidade Federal do Rio de Janeiro, RJ, Brasil. 


\section{INTRODUÇÃO}

As reservas de petróleo encontradas na camada pré-sal do litoral brasileiro representam um cenário promissor, porém repleto de desafios para a exploração e produção. Destacam-se as severas condições de processamento dos fluidos oriundos da rocha reservatório. Tais fluidos são tratados em Unidades de Produção, Armazenamento e Transferência, conhecidas por FPSO's (Floating Production, Storage and Offloading). Na separação da mistura de fluidos extraídos da rocha reservatório, os gases resultantes são devidamente tratados e armazenados e podem ser utilizados para completação de poços, como combustível para a FPSO ou ser transportado para uso em terra. Nesse ponto que emergem as características dos materiais envolvidos na operação. Com pressões em torno de 550 bar, temperaturas por volta de $-70^{\circ} \mathrm{C}$ e presença de $\mathrm{CO}_{2}$ e $\mathrm{H}_{2} \mathrm{~S}$, surge a necessidade de materiais que apresentem bom comportamento mecânico para dutos e outros componentes metálicos. Nestas condições, aços ou ligas devem apresentar boa resistência à corrosão, alta tenacidade em baixas temperaturas e elevada resistência mecânica [1].

Indicados inicialmente para aplicações criogênicas, como armazenamento de Gás Natural Liquefeito (GNL), os aços 9\% níquel possuem duas especificações de acordo com as normas ASTM A353 (dupla normalização e revenimento) e ASTM A553 Tipo 1 (têmpera e revenimento), sendo a norma ASTM A522 Tipo 1 indicada para componentes forjados, válvulas e flanges [2, 3, 4].

Tabela 1. Composição química $[2,3,4]$.

\begin{tabular}{ccc}
\hline Elemento (\%) & A353 \& A553 (Tipo 1) & A522 (Tipo 1) \\
\hline Carbono (máx.) & 0.13 & 0,13 \\
\hline Manganês (máx.) & 0.90 & 0,9 \\
\hline Fósforo (máx.) & 0.035 & 0,025 \\
\hline Enxofre (máx.) & 0.035 & 0,025 \\
\hline Silício & $0.15 / 0.40$ & $0,15 / 0,30$ \\
\hline Níquel & $8.50 / 9.50$ & $8,50 / 9,50$
\end{tabular}

As variações de propriedades mecânicas e metalúrgicas são oriundas de microestruturas diversificadas. Estas, por sua vez, são determinadas pelos tratamentos térmicos ou termomecânicos aplicados. No caso dos aços $9 \%$ níquel, a presença da estrutura austenítica oriunda do tratamento térmico é determinante para as propriedades mecânicas. A fase austenita uma vez formada pode se estabilizar durante o resfriamento [5].

Nas condições de operação citadas anteriormente temos a presença de $\mathrm{CO}_{2} \mathrm{e}$ $\mathrm{H}_{2} \mathrm{~S}$ em altas pressão e concentração os quais geram hidrogênio que pode se difundir, causando tensões internas inadequadas à estabilidade do material [6].

O hidrogênio na microestrutura de materiais metálicos tende a ser aprisionado em diversos tipos de imperfeições na rede cristalina como, contornos de grão, interfaces precipitado-matriz, discordâncias, lacunas, vazios, etc. A microestrutura dos aços é determinante neste sentido, dado que determinará a solubilidade, transporte e aprisionamento do hidrogênio na rede. $O$ aprisionamento de hidrogênio pode promover sua segregação, aumentando sua concentração local e favorecendo mecanismos de fragilização [5].

Os aprisionadores do hidrogênio podem se dividir quanto a energia de ligação em fracos, intermediários e fortes. Aprisionadores fracos possuem aproximadamente $20 \mathrm{KJ} / \mathrm{mol}$ de energia de ligação, estando neste grupo as discordâncias, precipitados 
finos e contornos de grão de baixo ângulo. Já aqueles intermediários possuem energia de ligação da ordem dos $50 \mathrm{~kJ} / \mathrm{mol}$; são microcavidades, as interfaces de ripas de martensita e os contornos de grãos da austenita prévia. Aqueles aprisionadores ditos fortes têm energias de ligação de $100 \mathrm{~kJ} / \mathrm{mol}$ a $120 \mathrm{~kJ} / \mathrm{mol}$; são precipitados esféricos e carbetos como $\mathrm{NbC}$, TiC ou MnS. Figuram também neste grupo as interfaces entre ripas de martensita, contornos de grão austeníticos com austenita retida, finos precipitados ou impurezas segregadas [5].

Neste sentido, o ensaio de Espectroscopia de Dessorção Térmica (TDS) destaca-se como forma de se caracterizar os sítios aprisionadores de hidrogênio. Após saturar o material de hidrogênio, a realização de aquecimento a uma taxa constante promove a dessorção gradual do mesmo, segundo a energia/temperatura referente a cada sítio aprisionador de sua microestrutura [7].

O presente trabalho tem por objetivo avaliar a fragilização por hidrogênio em aços $9 \%$ níquel com diferentes microestruturas através da identificação os seus sítios aprisionadores de hidrogênio.

\section{MATERIAIS E MÉTODOS}

Os materiais estudados consistem uma placa de aço 9Ni A553 como recebida e um tubo de aço $9 \mathrm{Ni}$ A522 tipo I nas condições como recebido e após tratamento térmico.

\subsection{Tratamentos térmicos (TT)}

Amostras do aço A522 foram submetidas a dois tratamentos térmicos diferentes com 0 intuito de obter diferentes frações de fase das microestruturas típicas para este aço para determinar posteriormente a influência dessas fases no aprisionamento de hidrogênio. O tratamento térmico foi realizado a $520^{\circ} \mathrm{C}$ por $1 \mathrm{~h}$, seguido de resfriamento ao ar.

\subsection{Preparação de Amostras}

A preparação metalográfica para a realização da caracterização microestrutural e hidrogenação eletrolítica consistiu em corte de precisão, desbaste com lixas de SiC de 200 a 2500 e polimento com pasta de diamante em panos de 3 e $1 \mu \mathrm{m}$.

\subsection{Caracterização Microestrutural - Microscopia Eletrônica de Varredura (MEV) \& Difração de Raios-X (DRX)}

A caracterização microestrutural dos materiais na condição como recebido e após tratamento térmico foi realizada através de microscopia eletrônica de varredura, utilizando um microscópio JEOL JSM 6460 com EDS Thermo/Noran System SIX - Model 200 acoplado.

A identificação das fases foi realizada através de ensaios de difração de raios$X$ para o aço A553 em um Shimadzu modelo XRD-6000 (PEMM), com os seguintes parâmetros: comprimento de onda $\lambda=1,5418 \AA$, faixa de varredura contínua de $10^{\circ}$ a $90^{\circ}$, passo $0,02^{\circ}$ e tempo $2^{\circ} / \mathrm{min}$. 


\subsection{Espectroscopia de Dessorção Térmica (TDS)}

Para a identificação dos aprisionadores de hidrogênio e suas respectivas energias, amostras do aço A553 foram hidrogenadas em solução de $\mathrm{NaOH} 0,1 \mathrm{M}$ sob uma corrente catódica de $60 \mathrm{~mA}$, por 48 horas, a temperatura ambiente. Já as do aço A522 foram hidrogenadas em solução $\mathrm{NaCl} 3,5 \%$, corrente catódica de 20 $\mathrm{mA}$, por 24 horas, também a temperatura ambiente.

Posteriormente à etapa de hidrogenação, ensaios de TDS foram realizados em um equipamento desenvolvido pelo Laboratório de Propriedades Mecânicas. Dentro de um reator de quartzo, sob um fluxo contínuo de hélio de alta pureza atuando como gás de arraste, as amostras foram aquecidas a partir da temperatura ambiente até aproximadamente $800^{\circ} \mathrm{C}$, sob taxas de aquecimento de $3,5,6,10$ e $15^{\circ} \mathrm{C} / \mathrm{min}$. O hidrogênio dessorvido foi detectado através de um espectrômetro de massa quadrupolar QMS 200 PrismaTM 80. A figura 1 esquematiza o aparelhamento do ensaio.

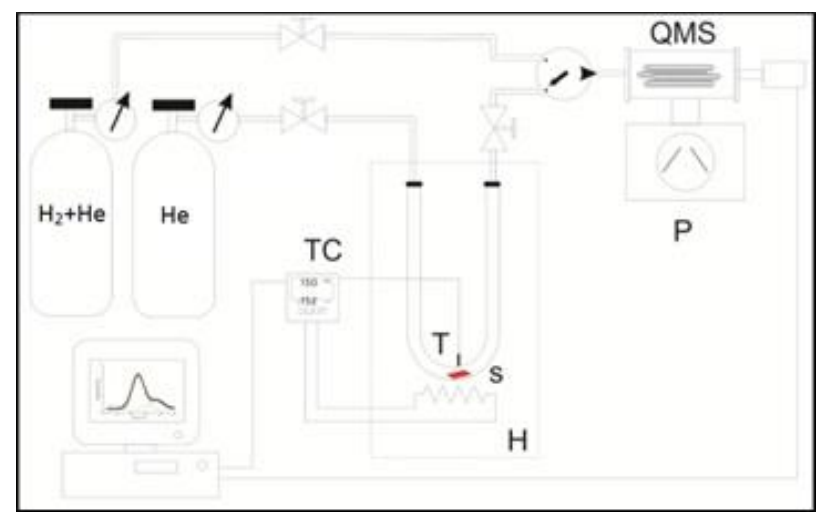

Figura 1. Esquema de ensaio de TDS: TC - controlador, T - termopar, $\mathrm{P}$ - bomba turbomolecular, QMS - espectrômetro de massa quadrupolar, $\mathrm{H}$ - forno, $\mathrm{S}$ - amostra; adaptado de [5].

\subsection{Metodologia para o Cálculo da Energia de Ativação para a Dessorção do Hidrogênio através do Espectro de TDS}

A fim de se calcular as energias associadas à dessorção do hidrogênio, foram utilizadas as temperaturas associadas aos picos nos espectros de TDS, $\mathrm{T}_{\mathrm{m}}$, e as taxas de aquecimento aplicadas, $\phi$.

Com base nas equações de KISSINGER [8], CHOO \& LEE [9] propuseram que as energias para ativação da dessorção dos sítios aprisionadores seguiam a relação matemática descrita pela equação 1, onde a taxa de aquecimento é representada por $\phi$, a temperatura referente ao pico de dessorção é representada por $T_{m}, R$ é a constante universal dos gases, e $\mathrm{E}_{\mathrm{p}}$ é a energia necessária à dessorção.

$$
\frac{\partial \ln \frac{\phi}{T_{m}^{2}}}{\partial \frac{1}{T_{m}}}=-\frac{E_{p}}{R}
$$

Ao se realizar ensaios a taxas diferentes com amostras semelhantes, é possível obter através do coeficiente angular da reta $\ln \frac{\phi}{T_{m}{ }^{2}}$ vs. $T_{m}$ a energia de dessorção associada a cada sítio; ou seja, cada sítio aprisionador irá gerar uma reta [9]. 


\section{RESULTADOS}

\subsection{Caracterização Microestrutural - Microscopia Eletrônica de Varredura (MEV) \& Difração de Raios-X (DRX).}

A figura 2(a) apresenta as imagens obtidas por MEV do aço A533 na condição como recebido. A microestrutura consiste em uma matriz martensítica/bainítica e partículas claras de austenita nos contornos. Essa microestrutura é semelhante àquela descrita por ZEEMANN e EMYGDIO [10]. Também foi possível observar faixas ou bandas de segregação (figura 2 - b), também observadas por QUEIROZ [11].

A figura 3(a) apresenta a microestrutura do aço 9Ni A522 tipo I na condição como recebido e as figuras $3(\mathrm{~b})$ e 3 (c) na condição tratado termicamente a $520^{\circ} \mathrm{C}$ por $1 \mathrm{~h}$ e a $580^{\circ} \mathrm{C}$ por $3 \mathrm{~h}$, respectivamente. Segundo a morfologia apresentada, a matriz é composta por martensita/bainita revenida, sendo a fase clara referente a austenita retida, como constatado também por QUEIROZ [11]. Observando as imagens não é possível perceber grandes variações microestruturais em relação a microestrutura como recebida. O tratamento realizado pode ser considerado um recozimento para alívio de tensões, como o que é feito após a soldagem. Uma possível variação na proporção de austenita retida será avaliada por difração de raios-X.

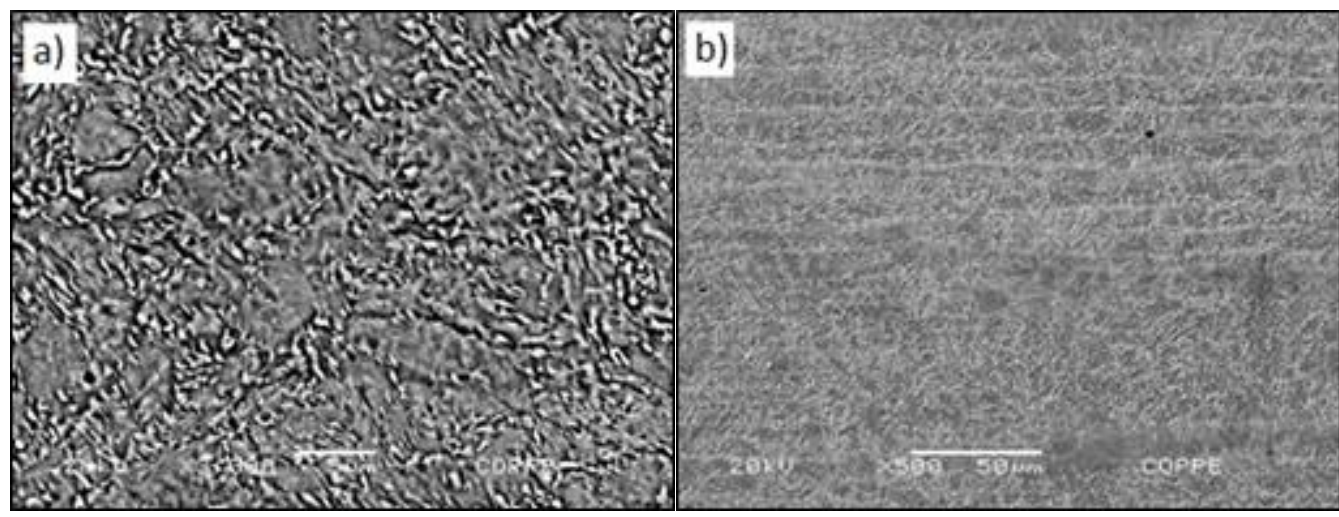

Figura 2. Microestrutura do aço A553 como recebido: a) elétrons retroespalhados permitem a visualização das "ilhas" de austenita retida, em tons claros; b) elétrons secundários permitem a visualização de bandas de segregação.

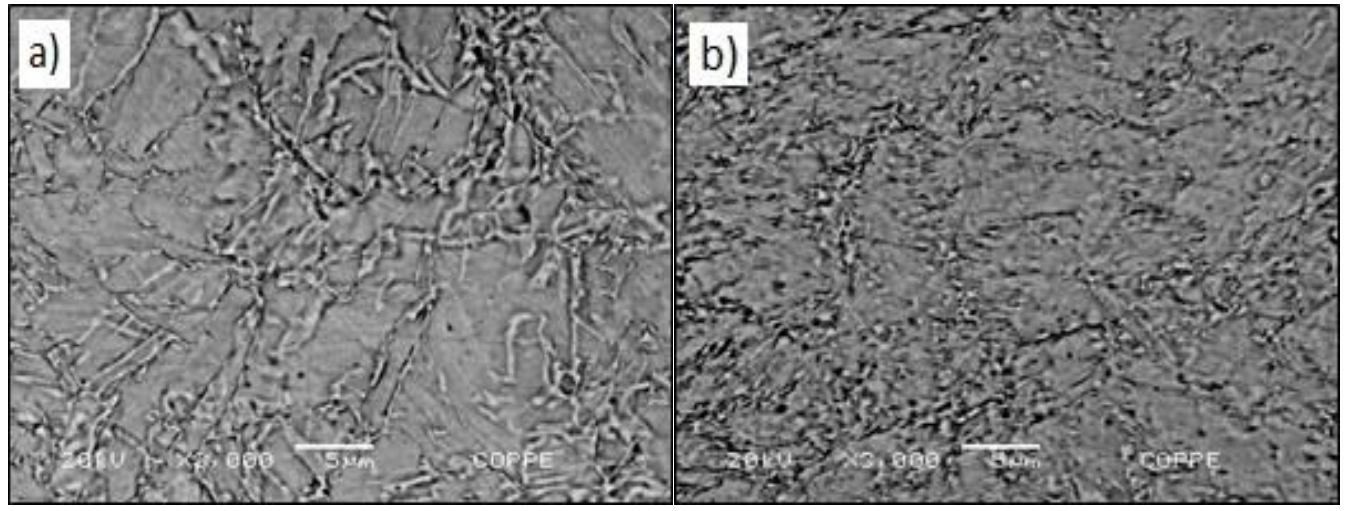




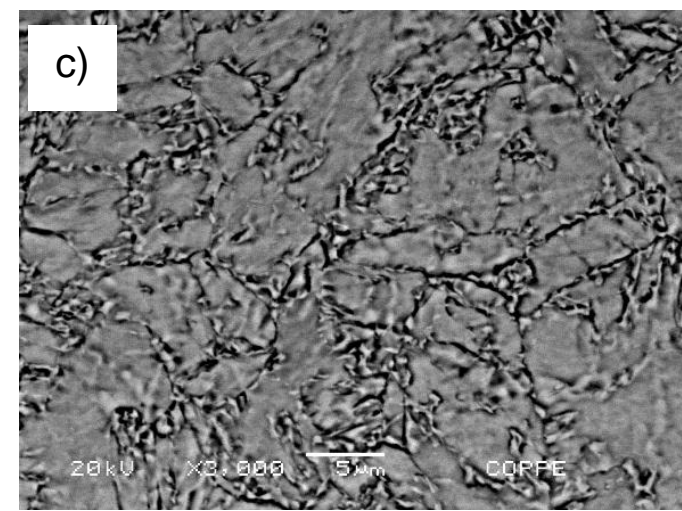

Figura 3. Microestrutura do aço A522: a) como recebido; b) TT a $520^{\circ} \mathrm{C}$ por $1 \mathrm{~h}$ e c) TT $580^{\circ} \mathrm{C}$ por $3 \mathrm{~h}$.

O difratograma observado na figura 4 para o aço A553 como recebido mostra a existência dos picos característicos às fases bainita ( $F e-\alpha, C C C)$, martensita ( $M$, TCC), e austenita (Fe-y, CFC), corroborando com as morfologias identificadas nas imagens de MEV e estudos realizados por QUEIROZ [11].

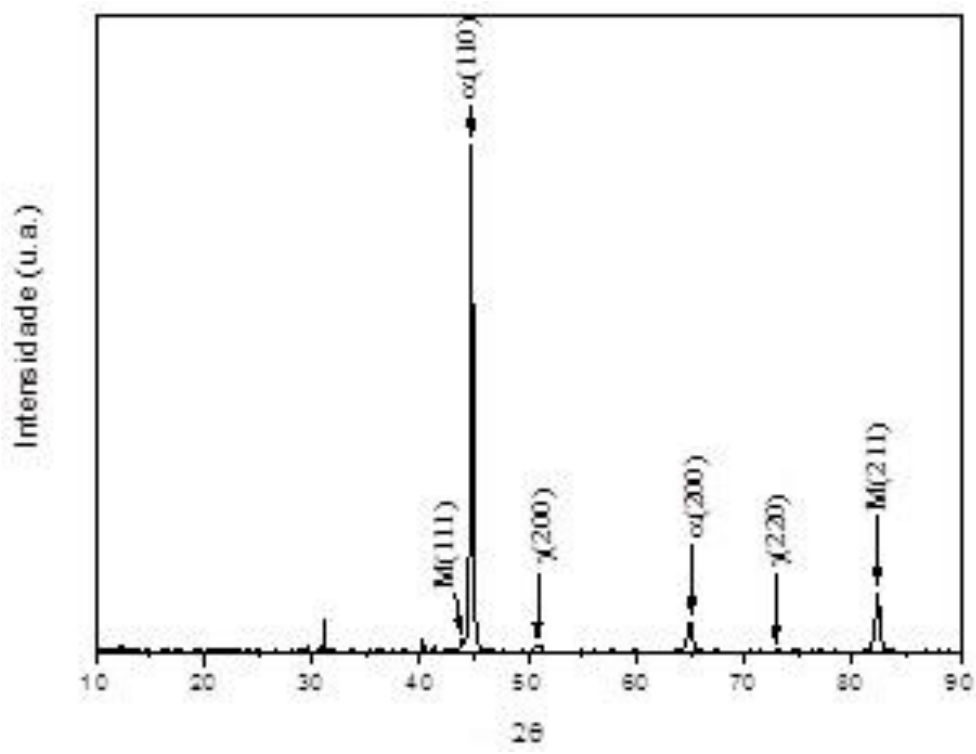

Figura 4. DRX do aço ASTM A553 como recebido, com orientação dos planos cristalográficos.

\subsection{Espectroscopia de Dessorção Térmica (TDS).}

Para identificar o número de picos existentes em cada espectro de dessorção do hidrogênio, foram realizadas deconvoluções Gaussianas, conforme pode ser observado no exemplo presente na figura 5(c). A ferramenta Peak Analyzer, utilizada para este fim, é disponibilizada no software OriginPRO 8.5. No entanto, não foi possível a realização de deconvoluções em alguns dos espectros, pelo motivo de não apresentarem iterações suficientes, pois os picos observados se mostravam muito próximos.

Nas figuras $5(\mathrm{a}), 5(\mathrm{~b})$ e $5(\mathrm{c})$, os espectros de dessorção do hidrogênio são referentes às taxas de aquecimento de 5,10 e $15^{\circ} \mathrm{C} / \mathrm{min}$, respectivamente, para 0 aço A553. A espectroscopia observada na figura $5(\mathrm{a})$, realizada a $5^{\circ} \mathrm{C} / \mathrm{min}$, apresentou um único pico de dessorção a $634^{\circ} \mathrm{C}$. A figura $5(\mathrm{~b})$ mostra um pico de dessorção a $657^{\circ} \mathrm{C}$ e refere-se àquele ensaio realizado a $10^{\circ} \mathrm{C} / \mathrm{min}$. Por fim, a figura 
5(c), para a dessorção realizada a $15^{\circ} \mathrm{C} / \mathrm{min}$, apresenta dois picos de dessorção sobreposto, a 591 e $684^{\circ} \mathrm{C}$.

Nas figuras $5(\mathrm{~d}), 5(\mathrm{e})$ e $5(\mathrm{f})$, os espectros de dessorção do hidrogênio são referentes às taxas de aquecimento de $3,6 \mathrm{e} 10^{\circ} \mathrm{C} / \mathrm{min}$, respectivamente, para o aço A522. A figura $5(\mathrm{~d})$ refere-se ao ensaio de TDS realizado a $3^{\circ} \mathrm{C} / \mathrm{min}$. Este espectro de dessorção do hidrogênio apresentou um pico a $530^{\circ} \mathrm{C}$ e outro a $657^{\circ} \mathrm{C}$. Já a figura $5(\mathrm{e})$, referente ao ensaio realizado a $6^{\circ} \mathrm{C} / \mathrm{min}$, indica a presença de três picos de dessorção, a 459,653 e $739^{\circ} \mathrm{C}$. Por fim, a figura $5(\mathrm{f})$ apresenta dois picos no espectro de dessorção do hidrogênio para a taxa de


Figura 5. Curvas de TDS para o aço $A 553$ como recebido a: a) $5^{\circ} \mathrm{C} / \mathrm{min}$, b) $10^{\circ} \mathrm{C} / \mathrm{min}$, e c) $15^{\circ} \mathrm{C} / \mathrm{min}$; e curvas de TDS para o aço A522 TT a: d) $3^{\circ} \mathrm{C} / \mathrm{min}$, e) $6^{\circ} \mathrm{C} / \mathrm{min}$, e f) $10^{\circ} \mathrm{C} / \mathrm{min}$. As temperaturas indicadas se referem aos picos de dessorção do hidrogênio dos aprisionadores na rede cristalina. 


\subsection{Cálculo da Energia de Ativação para a Dessorção do Hidrogênio}

A tabela 2 apresenta a relação de picos de dessorção do hidrogênio observados para o aço 9\% níquel (A553 e A522) e suas referidas taxas de realização do ensaio de TDS. Através do gráfico observado na figura 6 , foi calculada a energia de ativação para a dessorção do hidrogênio, obtendo-se $124,66 \mathrm{KJ} / \mathrm{mol}$.

Essa energia condiz com a literatura, classificando o sítio aprisionador observado como um aprisionador forte, consistindo de interfaces de grãos austeníticos com austenita retida [5]. Os resultados para a energia do sitio aprisionador também são corroborados pelas imagens de MEV obtidas para ambas as classes de aço $9 \%$ níquel estudadas

Tabela 2. Taxas de aquecimento com respectivos picos de dessorção observados nos ensaios de

\begin{tabular}{cc}
\multicolumn{2}{c}{ TDS; ${ }^{*}$ A553, ${ }^{* *}$ A522. } \\
\hline $\boldsymbol{\phi}\left[{ }^{\circ} \mathbf{C} / \mathbf{m i n}\right]$ & Pico $\left[{ }^{\circ} \mathbf{C}\right]$ \\
\hline $3^{* *}$ & - \\
\hline $5^{*}$ & 634 \\
\hline $6^{* \star}$ & 641 \\
\hline $10^{*}$ & 657 \\
\hline $10^{* \star}$ & 676 \\
\hline $15^{*}$ & 684 \\
\hline $\boldsymbol{E}_{\boldsymbol{p}}[\mathrm{KJ} / \mathrm{mol}]$ & $\mathbf{1 2 4 , 6 6}$ \\
\hline
\end{tabular}

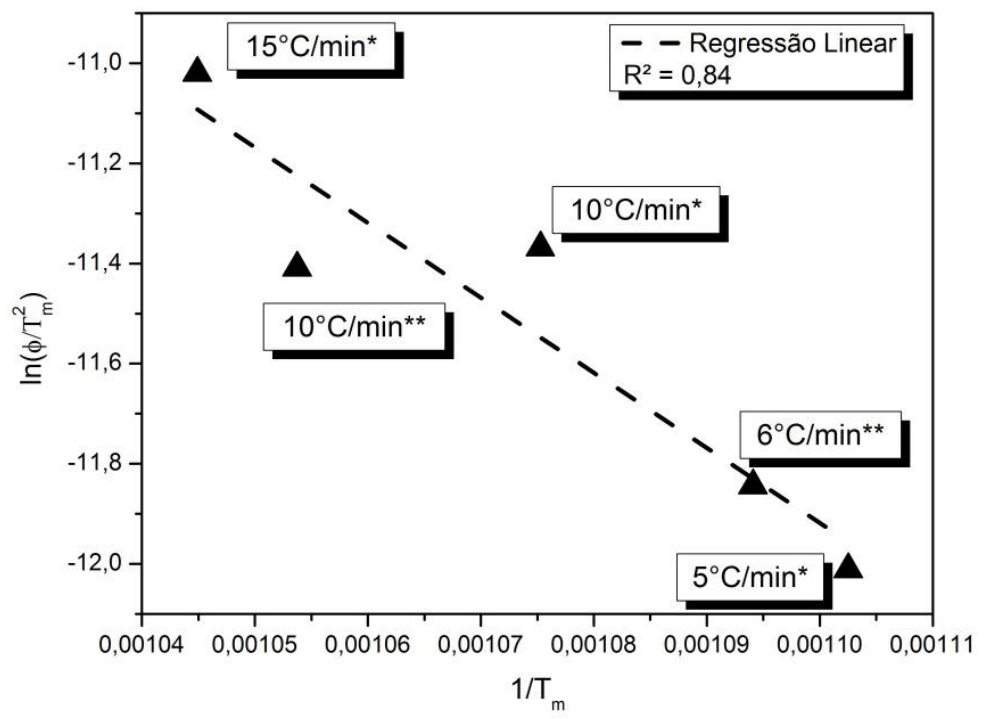

Figura 6. Gráfico referente à equação 1 para o aço $9 \mathrm{Ni} ;{ }^{*} \mathrm{~A} 553,{ }^{* *} \mathrm{~A} 522$ [9].

\section{CONCLUSÕES}

As imagens de MEV e DRX mostram que, para ambos os aços a microestrutura é composta de uma matriz martensítica/bainítica e austenita retida. $A$ quantidade de austenita retida muda de um aço para outro, mas aparentemente não houve mudança significativa em sua quantidade para os dois tratamentos térmicos propostos. A quantificação da austenita retida deve ser feita através do método Rietveld para determinar a sua influência no processo de fragilização pelo $\mathrm{H}$. 
A energia de ativação para a dessorção do hidrogênio observada $(124,66$ $\mathrm{KJ} / \mathrm{mol}$ ) determinou a presença de interfaces de grãos de austenita retida [5], classificando este como um forte aprisionador de hidrogênio para os aços $9 \%$ níquel.

\section{Agradecimentos}

Os autores agradecem ao Laboratório Multiusuário de Caracterização de Materiais, ao Núcleo Multiusuário de Microscopia e à FAPERJ pelo apoio financeiro à pesquisa.

\section{REFERÊNCIAS}

1 Petrobras, 2012, "Perspectiva de Aplicação de Aços ao Níquel em Sistemas de Processamento de CO2 para o Pré-Sal", Speech at Rio Oil \& Gas 2012 Conference, Rio de Janeiro, IBP.

2 ASTM, Standard specification for pressure vessel plates, alloy steel, double-normalized and temperade $9 \%$ nickel, reaprovada, 2014.

3 ASTM, Standard specification for pressure vessel Plates, alloy steel, quenched and Tempered 7, 8, and 9\% nickel, reaprovada, 2014.

4 ASTM, Standard specification for forged or rolled 8 and $9 \%$ nickel alloy steel flanges, fittings, valves, and parts for low-temperature service, reaprovada, 2014.

5 Torres, L. F. L., "Influência da Microestrutura na Interação do Hidrogênio com o Aço 2,25Cr-1Mo Envelhecido", Tese de D. Sc., Rio de Janeiro, RJ, Brasil, COPPE/UFRJ, 2011.

6 Silva, B.R.S., "Estudo da Fragilização pelo Hidrogênio em Juntas Soldadas de Tubos do Aço Inoxidável Super Duplex UNS S32750", Dissertação de M.Sc., Rio de Janeiro, RJ, Brasil, COPPE/UFRJ, 2015.

7 Sven L.M. Schroeder and Michael Gottfried, "Temperature-Programmed Desorption (TPD) \& Thermal Desorption Spectroscopy (TDS)", Advanced Physical Chemistry Laboratory, Berlim, 2002.

8 Kissinger, H., "Reactions kinectics in differential analisys," Analytical Chemistry, pp. 147-157, 1957.

9 Choo, J., Lee, W., "Thermal analysis of trapped hydrogen in pure iron," Metallurgical and Materials Transactions, pp. 135-140, 1982.

10 Zeemann, A., Emydgio, G., "9\% Ni Alloy Steel for H2S Service", CORROSION/2014, paper n. 4361, San Antonio, NACE.

11 Queiroz, V. F. M., "Estudo dos efetios da permeação do hidrogênio em aços 9Ni", Dissertação e M. Sc., Rio de Janeiro, RJ, Brasil, COPPE/UFRJ, 2016. 